Leth-Moller, 2016). Owing to its uncommon use, there is limited and conflicting data on the risk of hyponatremia with Moclobemide, a reversible inhibitor of monoamine oxidase A (Mercier, 1997; Mazhar, 2019). There are few reports of hyponatremia induced by multiple antidepressant classes in the same patient.

OBJECTIVE: To add to the literature on risk of hyponatremia with Moclobemide and other antidepressants.

METHODS: We report a case of hyponatremia sequentially induced by multiple different antidepressant classes who was treated with Moclobemide with no recurrence of hyponatremia. We review existing literature on antidepressant-induced hyponatremia.

RESULTS: A 67-year-old man with a history of hypertension, dyslipidemia and gout was first diagnosed with major depressive disorder at age 50 after presenting with pervasive depressed mood, anhedonia, insomnia, poor concentration and feelings of worthlessness. Investigations found no medical causes of depression. His depression remitted on Venlafaxine $75 \mathrm{mg} /$ day with no hyponatremia induced. During a second depressive episode 4 years later, his serum sodium (Na) dropped from a normal baseline to $122 \mathrm{mmol} / \mathrm{L}$ after Venlafaxine was restarted. He appeared euvolemic on physical examination. Investigations found no other causes of hyponatremia and were consistent with SIADH, which was attributed to Venlafaxine. His depression later remitted on Mirtazapine $30 \mathrm{mg} /$ day with no hyponatremia induced. During his third depressive episode at age 67 , he developed hyponatremia (serum Na $123 \mathrm{mmol} / \mathrm{L}$ ) a week after restarting Mirtazapine. His clinical picture was consistent with SIADH. He later developed hyponatremia after initiating the following antidepressants sequentially: Fluvoxamine, Agomelatine, Nortriptyline, Bupropion. Hyponatremia resolved with fluid restriction and cessation of the implicated antidepressant each time before the next was initiated. He eventually tolerated Moclobemide $300 \mathrm{mg} /$ day with no recurrence of hyponatremia.

CONCLUSIONS: Agomelatine, Nortriptyline and Bupropion are reported to have a low risk of hyponatremia but were implicated in this case. Venlafaxine and Mirtazapine did not cause hyponatremia when first taken but were implicated when restarted after a period of cessation, underscoring the idiosyncratic nature of antidepressantinduced hyponatremia. Moclobemide can be considered for depressed patients with recurrent antidepressantinduced hyponatremia. Serum Na should be regularly monitored in patients taking antidepressants who are at high risk of hyponatremia.
128

\section{Factors Associated with Cost Savings Following Use of a Pharmacogenetic Assay in Individuals with Mood and Anxiety Disorders}

Alison MEdwards, MStat ${ }^{\prime}$; Roy H Perlis, MD, MSc ${ }^{2}$; and David $S$ Krause, $M D^{3}$

${ }^{1}$ Healthagen, New York, NY

${ }^{2}$ Massachusetts General Hospital and Harvard Medical

School, Boston, MA

${ }^{3}$ Genomind, King of Prussia, PA

ABSTRACT: Background: In a study conducted in the database of a large commercial healthcare insurer, we previously demonstrated that use of a commercial pharmacogenetic assay for individuals with mood disorders was associated with decreased resource utilization and cost in the 6 month period following use compared to propensity-score matched controls. We conducted a post hoc analysis to understand variables associated with high cost savings.

METHODS: The results and methods of the initial study have previously been described. Cases were individuals with mood and anxiety disorders who received a commercial pharmacogenetic assay (Genomind, King of Prussia PA) to inform pharmacotherapy. 817 tested individuals (cases) with mood and/or anxiety disorders were matched to 2745 controls. Overall costs were estimated to be $\$ 1,948$ lower in the tested group. The differences were largely the result of lesser emergency room and inpatient utilization for cases. In the present analysis, cost difference for cases compared to their matched controls was rank ordered by decile. High cost savers were arbitrarily defined a priori as the top $20 \%$ of savers. Using multivariable modeling techniques, an ordinal logistic regression model was generated in which baseline or follow-up variables were statistically tested for independent associations with high, low, and no cost savings.

RESULTS: 606 (74\%) of cases were net cost savers compared to their controls (cost difference $<0$ ). High cost savers $(n=121)$ saved on average $\$ 10,690$ compared to their matched controls. They were statistically more likely to have been diagnosed with bipolar disorder $(n=33 / 121)$ than low cost savers $(n=57 / 485)$ or non-savers $(n=31 / 211)$, and had a lower Charlson Comorbidity index. High cost savers had fewer mean number of antidepressants in the baseline period (mean=3.16) compared to non-savers (3.73) but more than low cost savers $(2.72)(\mathrm{p}<0.05$ across groups). In a multivariable model, bipolar, count of antidepressants, outpatient visits, and inpatient visits were statistically associated with being a high cost saver; antidepressant count and all-cause inpatient and outpatient visits in the baseline period were inversely associated with cost savings. 
CONCLUSIONS: Use of a pharmacogenetic assay was associated with cost-savings in the database of a large commercial insurer. Patients with bipolar disorder were more likely to be high cost savers than individuals with other mood and anxiety disorders.

Funding Acknowledgements: Genomind

129

\section{Medication Assisted Treatment Protocol}

David Michael Mathis, DO FASAM DABAM

ABSTRACT: This article describes a protocol to be able to utilize medication assisted treatment options for patients dependent on opioids. The first step is using a 15-day Klonopin taper for effect detox of acute opioid withdrawal. Once detoxed, the patient can be started on low dose methadone or low dose Buprenorphine. Titration above $40 \mathrm{mg}$ of Methadone, or $8 \mathrm{mg}$ of buprenorphine will usually not be needed. Buprenorphine is utilized as the mono product, Subutex. Avoiding Suboxone eliminates the risk of reemergence of acute opioid withdrawal symptoms.

A description of how to transition to Naltrexone is provided. There are some differences between Methadone and Buprenorphine in the transition to Naltrexone. Once the patient is transitioned to Naltrexone, the stage is set for the patient to be able to get off medication assisted treatment.

\section{0}

Temporal Lobe Epilepsy: Is It in Your Differential Diagnosis? Two Case Reports

Debra $K$ Avery, PMHNP-BC, $B S A^{\prime}$; and Julie Brann D. Min., PMHNP-BC'

${ }^{1}$ Independent Practitioner, Colorado Springs, CO

ABSTRACT: Temporal Lobe Epilepsy: Is it in your differential diagnosis? Two Case Reports

OBJECTIVE: Temporal Lobe Epilepsy (TLE), also referred as Complex Partial Seizures, is a medical diagnosis that must be considered in the pediatric, adolescent, and adult population presenting for psychiatric care. Mood disorders are common in people with epilepsy, with a prevalence rate of 20 to $50 \%$. Scant literature exists that seeks specifically to enhance our knowledge of the similarities and subtle differences between TLE, Bipolar Disorder (BD) and Post Traumatic Stress Disorder (PTSD). Our objective is to describe an adolescent and adult case; one initially diagnosed as BD, the other PTSD, when in fact, it was TLE. We aim to illustrate that misdiagnosis and failure to diagnose are common. The provider must engage in a thorough review of systems and consider
TLE in the differential diagnosis. A delay in the appropriate diagnosis and treatment can lead to a substantial amount of adverse effects and worsening of symptoms and negatively impact one's quality of life.

METHOD: Two case studies; an adolescent and one adult, diagnosed with BD and the other PTSD. Both endorsed a history of symptoms indicative of TLE. Key assessment findings and screening diagnostics alerted us to the differential diagnosis of TLE. The overlap of the symptom presentation is described.

RESULTS: TLE and many psychiatric conditions often present with overlapping symptoms. Patients have the potential to present with absence seizures, unprovoked irritability, oppositionality, aggression, anger, paroxysmal anxiety, somatic symptoms such as headaches, nausea, burning in the abdomen, stereotyped movements or behaviors, hypergraphia bizarre or incongruous affect, symptoms of fear, disturbed sleep, tearfulness, memory problems, déjà vu, fugue states, changes in cognition, inability to concentrate, fatigue, auditory and visual hallucination and bad temper. Our differential diagnosis of TLE was confirmed with electroencephalogram (EEG). By prescribing the appropriate medications to these two individuals, they were able to experience improved moods, become more productive in society, working, attending church, family outings, etc. They were weaned off their antipsychotic medications, of which an abundance of troubling side effects is now a non-issue.

CONCLUSIONS: A delay in the proper diagnosis of TLE can have a significant negative impact on the adolescent and adult population. A need exists to educate mental health professionals on the overlap of symptoms of TLE and psychiatric disorders. The significant issue at hand is that they may not be receiving adequate or appropriate medications. Considering TLE in the differential diagnosis of presenting mood instability ensures our patients they are getting the basics of psychiatric care; which always emphasizes ruling out medical conditions first.

131

\section{A Marionettist Pulling My Strings: A Case of Buprenorphine-induced Chorea}

\author{
Dev Patel ${ }^{\prime, 2}$; Ishandeep Gandhi'; Faisal Malek'; \\ Camille Olechowski $i^{3}$ and Alan R. Hirsch ${ }^{4}$ \\ ${ }^{1}$ Aureus University School of Medicine, Oranjestad, Aruba \\ ${ }^{2}$ Illinois Center for Neurological and Behavioral \\ Medicine, Des Plaines, Illinois \\ ${ }^{3}$ Trinity School of Medicine, St. Vincent and The \\ Grenadines \\ ${ }^{4}$ Illinois Center for Neurological and Behavioral \\ Medicine, Des Plaines, Illinois.
}

